\title{
Fidelity Concepts Used in UWB Systems
}

\author{
Mohsen Koohestani ${ }^{1,2}$, Antonio A. Moreira ${ }^{1}$, Anja K. Skrivervik ${ }^{2}$ \\ ${ }^{1}$ Instituto de Telecomunicações, Instituto Superior Técnico, Universidade Técnica de Lisboa, \\ Av. Rovisco Pais 1, 1049-001 Lisboa, Portugal \\ ${ }^{2}$ Laboratoire d'Electromagnétisme et d'Acoustique, École Polytechnique Fédérale de Lausanne, \\ STI-IEL, Station 11, CH-1015 Lausanne, Switzerland \\ koohestani.mohsen@epfl.ch, antonio.moreira@1x.it.pt, anja.skrivervik@epfl.ch
}

\begin{abstract}
Since its introduction the fidelity concept has been used to evaluate the time behavior of UWB antennas. However, fidelity has been employed with different meanings. This paper clarifies the differences between fidelity factor, system fidelity factor, and fidelity factor of the system. A recently developed UWB antenna has been taken as a representative one to illustrate the differences among these concepts.
\end{abstract}

\section{INTRODUCTION}

In 1994, Lamensdorf et al. introduced a parameter named fidelity to quantify the distortion between two time signals [1]. It has been defined as the correlation between two signals, normalized to their energies. The usefulness of this concept was discussed in that paper.

In our view, some UWB antenna published papers have used a fidelity parameter, corresponding to divergent definitions, to assess the antenna time behavior (see for instance [2]-[5]). The term has been employed referring to the correlation between the input signal derivatives and the signal radiated by the antenna, as studied in [6], or between the input and the received signals, as proposed in [7], or between the signal in different angular directions with respect to the main beam direction, as investigated in [8]. This paper aims to clarify the differences between these definitions by evaluating time signals correlations in the case of a recently developed UWB antenna [9], depicted in Fig. 1.

\section{SiMULATION AND MEASUREMENT SCENARIOS}

After the introduction of the fidelity concept, there has been direct and indirect ways to experimentally evaluate fidelity. This also led to the arising of some misuse of its definition. In this section the scenarios in which this parameter is simulated and measured, with regard to each definition, is presented.

\section{A. Fidelity Factor}

The original definition of the fidelity factor (FF) takes into account the effect of the transmitting antenna, only, and the correlation refers to the input signal derivatives and the signal radiated by the antenna under test [6], [10]-[11]. To evaluate this parameter, using CST, an ideal far-field linearly-polarized probe is taken to record the radiated signal of the transmitting antenna [12], and then the Matlab ${ }^{\mathrm{TM}}$ software package is used to

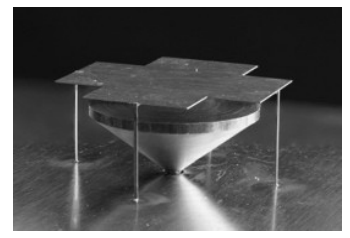

(a)

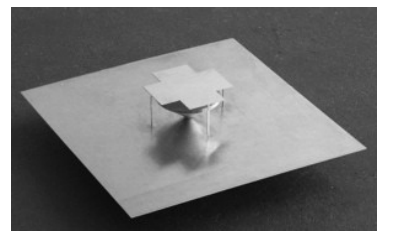

(b)
Figure 1. Top-cross-plate UWB mono-cone antenna [9]:

(a) close-view; and (b) full-view.

produce the differentiations of the input signal and to calculate the correlation [13]. In measurements, the transfer function of the receiving antenna and the cable effects should be removed, although the radio channel influence is present. This can be done by Matlab analysis using the method presented in [6]. Hence, any UWB receiving antenna type could be used. Usually, a FF greater than 0.9 is acceptable for pulse transmission [14].

\section{B. System Fidelity Factor}

Another definition, the system fidelity factor (SFF) introduced in [7], takes into account the distortion induced by both transmitting and receiving antennas and can be quantified through the analysis of the correlation between the input and the received signals. In this scenario, the radio channel is considered. In simulations, instead of an ideal probe, any UWB receiving antenna could be used. Consequently, SFF can be directly measured, in contrast to FF. A distortion higher than $50 \%(\mathrm{SFF}<0.5)$ leads to an almost unrecognizable pulse [7].

\section{Fidelity Factor of the System}

We distinguish the previous definition from another one, which we refer to as "fidelity factor of the system" (FFS), that describes the spatial radiation properties of an antenna. For a two antennas system the correlation is between the radiated signal which is received in an arbitrary angular direction and the signal in the main beam direction [8], [15]-[16]. This characterization doesn't indicate how much distortion the input signal experiences due to the radio channel. By rotating the receiving antenna to get the transmitted signal in other directions from a reference direction, this parameter can be calculated both in simulation and measurement scenarios. We assume the acceptable criterion for pulse distortion the same as for FF. 


\section{RESULTS AND DISCUSSION}

The FF, SFF, and FFS have been evaluated for a recently developed UWB antenna [9] in order to illustrate the differences in their definitions. All these parameters depend on the assumed pulse shape. Here, the $\mathrm{CST}^{\circledR}$ default Gaussian modulated pulse in the desired frequency band $(3.1-10.6 \mathrm{GHz})$ was used as the input pulse. This fully complies with FCC indoor and outdoor power masks [17]. For the FF calculation, the CST ideal field probes were placed at the far-field of the transmitting antenna to receive the radiated signals. The $0^{\text {th }}, 1^{\text {st }}$, $2^{\text {nd }}$ and $3^{\text {rd }}$ derivatives of the input pulse were obtained using Matlab and then the correlations between theses derivatives and the probe signal were calculated. The evaluated FF for three directions in the azimuthal plane $\left(\varphi=0^{\circ}, 30^{\circ}\right.$, and $\left.60^{\circ}\right)$ are given in Table I. The SFF was directly calculated from the correlation of the simulated input and received signals obtained from HFSS analysis [9]; results are shown in Table II. The time-domain setup is described in that paper. To determine the FFS, the correlation between the received signals in $0^{\circ}$ and the two other considered directions were calculated and presented in Table III. Results in Table I show that the radiated pulse has a higher correlation to the input pulse than the derivatives. It can be observed that effect of the differentiation of the transmitting antenna signal is small, as the FF value differences between the derivatives are minute. Based on the results shown in Table II, the SFF is always smaller than the FF (considering the $0^{\text {th }}$ and $1^{\text {st }}$ derivatives) as it accounts for the distortion introduced both at the transmit and the receive sides, as expected. Results in Table III show higher FFS values, which is also expected for the considered antenna as it radiates almost omni-directionally.

\section{TABLE I. FIDELITY FACTOR}

\begin{tabular}{c|c|c|c|c}
\multirow{2}{*}{$\varphi$} & \multicolumn{4}{|c}{$\mathrm{FF}$} \\
\cline { 2 - 5 } & $0^{\text {th }}$ derivative & $1^{\text {st }}$ derivative & $2^{\text {nd }}$ derivative & $3^{\text {rd }}$ derivative \\
\hline $0^{\circ}$ & 0.985 & 0.959 & 0.914 & 0.857 \\
\hline $30^{\circ}$ & 0.986 & 0.948 & 0.897 & 0.825 \\
\hline $60^{\circ}$ & 0.987 & 0.949 & 0.898 & 0.826
\end{tabular}

TABLE II. SyStem FidELITY FACTOR

\begin{tabular}{c|c}
$\varphi$ & $\mathrm{SFF}$ \\
\hline $0^{\circ}$ & 0.862 \\
\hline $30^{\circ}$ & 0.828 \\
\hline $60^{\circ}$ & 0.829
\end{tabular}

TABLE III. FIDELITY FACTOR OF THE SYSTEM

\begin{tabular}{c|c}
$\varphi$ & FFS \\
\hline $0^{\circ}$ & 1.000 \\
\hline $30^{\circ}$ & 0.963 \\
\hline $60^{\circ}$ & 0.960
\end{tabular}

\section{CONCLUSION}

The differences between the fidelity factor, system fidelity factor, and fidelity factor of the system have been investigated. The evaluation of the time signals correlations in the case of a recently developed UWB antenna were taken to clarify the differences. It was elucidated that the FF doesn't indicate the receiving antenna effects, whereas the FFS disregards the amount of distortion of the input signal. Moreover, the SFF takes into account the distortion induced by both transmitting and receiving antennas; hence, it would always have smaller values than FF and FFS.

\section{ACKNOWLEDGEMENT}

This work was supported by the Portuguese Fundação para a Ciência e a Tecnologia (FCT) project Pest-OE-EEI-LA00082011, under Grant SFRH/BD/51445/2011.

\section{REFERENCES}

[1] Lamensdorf, D.; Susman, L., "Baseband-pulse-antenna techniques," IEEE Antennas Propag. Magazine, vol. 36, no. 1, pp. 20-30, Feb. 1994.

[2] Tokan, N.T; Neto, A.; Tokan, F.; Cavallo, D., "Comparative study on pulse distortion and phase aberration of directive ultra-wideband antennas," IET Microw. Antennas \& Propag., vol. 7, no. 12, pp. 10211026, 2013.

[3] Koohestani, M.; Pires, N.; Skrivervik, A.K.; Moreira, A.A., "Influence of dielectric loading on the fidelity factor of an Ultra Wideband monopole antenna," Antennas and Propagation Conference (LAPC), Loughborough, GB, pp. 1-3, 2012.

[4] Akbari, M.; Zarbakhsh, S.; Marbouti, M., "A novel UWB antenna with dual-stopband characteristics," Microw. and Opt. Technol. Lett., vol. 55, no. 11, pp. 2350-2354, 2013.

[5] Shameena, V.A.; Mridula, S.; Pradeep, A.; Jacob, S.; Lindo, A.O.; Mohanan, P.; "A compact CPW fed slot antenna for ultra wide band applications," Elsevier AEU International Journal of Electronics and Communications, vol. 66, no. 3, pp. 189-194, 2012.

[6] Dumoulin, A.; John, M.; McEvoy, P.; Ammann, M.J., “An enhanced method to measure pulse disperion in UWB antennas," IEEE Antennas Propag. Society Inter. Symp. (APS/URSI), pp. 1-2, 2012.

[7] Quintero, G.; Zürcher, J.-F.; Skrivervik, A. K.; "System fidelity factor: A new method for comparing UWB antennas," IEEE Trans. Antennas Propag., vol. 59, no. 7, pp. 2502-2512, 2011.

[8] Alomainy, A.; Sani, A.; Rahman, A.; Santas, J. G.; Hao, Y., "Transient characteristics of wearable antennas and radio propagation channels for ultrawideband body-centric wireless communications," IEEE Trans. Antennas Propag., vol. 57, no. 4, pp. 875-884, 2009.

[9] Koohestani, M.; Zürcher, J.-F.; Moreira, A.A., Skrivervik, A.K., "A novel, low-profile, vertically-polarized UWB antenna for WBAN," IEEE Trans. Antennas Propag., Minor revision, 2013.

[10] Ghosh, S., "Band-notched modified circular ring monopole antenna for ultrawideband applications," IEEE Antennas Wirel. Propag. Lett., vol. 9, pp. 276-279, 2010.

[11] Elmansouri, M.A.; Radway, M.J.; Filipovic, D.S., "Frequency- and timedomain performance of four-arm mode-2 spiral antennas," IEEE Trans. Antennas Propag., vol. 60, no. 6, pp. 2627-2634, 2012.

[12] CST commercial software package. Available online at www.cst.com.

[13] MATLAB version 7.14. Natick, Massachusetts: The MathWorks Inc., 2012. Available online at www.mathworks.com.

[14] Fallahi, H.; Atlasbaf, Z., "Study of a class of UWB CPW-fed monopole antenna with fractal elements," IEEE Antennas Wirel. Propag. Lett., vol. 12, pp. 1484-1487, 2013.

[15] Pancera, E.; Zwick, T.; Wiesbeck, W., "Spherical Fidelity Patterns of UWB Antennas," IEEE Trans. Antennas Propag., vol. 59, no. 6, pp. 2111-2119, 2011.

[16] Guo-Ping Gao; Bin Hu; Jin-Sheng Zhang, "Design of a miniaturization printed circular-slot UWB antenna by the half-cutting method," IEEE Antennas and Wirel. Propag. Lett., vol. 12, pp. 567-570, 2013

[17] Koohestani, M.; Pires, N.; Skrivervik, A.K.; Moreira, A.A., "Timedomain performance of patch-loaded band-reject UWB antenna," Electronics Letters, vol. 49, no. 6, pp. 385-386, 2013. 